\title{
Optimized Polarization Build-Up Times in Dissolution DNP-NMR Using a Benzyl Amino Derivative of BDPA.
}

\author{
J. L. Muñoz-Gómez, ${ }^{a, \neq}$ E. Monteagudo, ${ }^{b, \sharp}$ V. Lloveras, ${ }^{a}$ T. Parella, ${ }^{b}$ J. Veciana, ${ }^{a, *}$ and J. Vidal-Gancedo ${ }^{a, *}$
}

The synthesis of two novel BDPA-like radicals, a benzyl amino (BAm-BDPA, 7) and a cyano (CN-BDPA, 5) derivatives, is reported and their behaviour as polarizing agents for fast dissolution Dynamic Nuclear Polarization (DNP) is evaluated. The radical 7 is a promising candidate for DNP studies since it is soluble in neat $\left[1-{ }^{13} \mathrm{C}\right]$ pyruvic acid (PA), and therefore the use of an additional glassing agent for sample homogeneity is avoided. In addition, a $60 \mathrm{mM}$ sample of 7 offers optimum ${ }^{13} \mathrm{C}$ NMR signal enhancements by using fairly short polarization times (about 1800 s). It is shown that DNP-NMR measurements using 7 can be performed much efficiently in terms of signal enhancement by polarization build-up time unit than using the reference OX63 or BDPA radicals. These enhanced features are translated to a substantial reduction of polarization times that represent an optimum temporary use of the DNP polarizer and allow economize liquid helium consumption.

\section{Introduction}

Nuclear Magnetic Resonance (NMR) spectroscopy is an essential analytical technique widely used for the structural characterization and quantification of chemical compounds. On the other hand, Magnetic Resonance Imaging (MRI) has become a very important non-invasive tool in medicine for early diagnostic of many diseases; for instance to obtain metabolic maps, anatomical and functional information, flux perfusion and diffusion studies, among others. ${ }^{1}$ The main drawback of NMR/MRI techniques is their poor sensitivities due to the inherent low thermal-equilibrium Boltzmann polarization of some interesting nuclear spins, such as the low natural-abundance ${ }^{13} \mathrm{C}$ and ${ }^{15} \mathrm{~N}$. Different hyperpolarization methods ${ }^{2}$ have been developed to enhance the NMR signal, such as optical pumping, ${ }^{3,4}$ para-hydrogen induced polarization ${ }^{5,6}$ (PHIP) and dynamic nuclear polarization $^{7,8,9}$ (DNP). Among them, dissolution DNP has become a powerful methodology for obtaining strongly polarized solutions for its use in both in-vitro and in-vivo applications; ${ }^{10}$ for structural elucidation, ${ }^{11,12}$ to monitor chemical and biochemical reactions, ${ }^{13,14}$ to study molecular interactions, ${ }^{15}$ to develop new and ultrafast NMR experiments, ${ }^{16,17}$ to perform in-vivo ${ }^{13} \mathrm{C}$ imaging of metabolic reactions ${ }^{10,18}$ or looking for new biomarkers by real-time molecular imaging. ${ }^{19,20}$ Recently, dissolution DNP has allowed real time assessment of low sensitive nuclei such as ${ }^{13} \mathrm{C}$ by in-vivo $\mathrm{NMR}^{21}$ and the development of new $\mathrm{pH}$-sensitive MRI agents. ${ }^{22}$ DNP experiments involve a sample containing the analyte, a source of free electrons, typically a radical molecule, and often a glassing agent solvent to ensure the sample homogeneity, leading the proper polarization transfer from the unpaired electrons of the organic radical to the nearby nuclei of the analyte. Radicals such as TEMPO, ${ }^{23} \mathrm{BDPA},{ }^{24}$ or trityl, ${ }^{25}$ have been widely used as mono- and/or biradicals as DNP polarizing agents. ${ }^{26}$ Nowadays, the most extended radical used for in-vivo DNP applications is the trityl-based stable free radical OX63, ${ }^{27}$ mainly because it presents a narrow electron paramagnetic resonance (EPR) linewidth which enable to polarize low- $\gamma$ nuclei. However, the OX63 radical is a very expensive commercial compound and soluble in the most common buffered solution conditions, hampering the translation of its hyperpolarizing features into human subject experiments. Additionally, it presents relatively long polarization build-up times (typically 60-90 min.), with the consequent time-consuming measurements and high helium consumption.

Recently, the carbon centered BDPA radical, ${ }^{24}$ (8 in Chart 1) and the benzyl alcohol derivative of BDPA (BA-BDPA) ${ }^{28}$ have been reported as new feasibly polarizing agents for dissolution DNP. The original radical $\mathbf{8}$, which has a comparable EPR linewidth to OX63, is a versatile polarizing agent under a variety of sample conditions, ${ }^{29}$ from solid-state $\mathrm{DNP}^{30}$ to fast dissolution DNP of [1${ }^{13}$ C]pyruvic acid (PA). ${ }^{31}$ In the latter case, although the residual BDPA radical traces can be removed from the hyperpolarized solution by filtration, its insolubility into neat PA requires the use of sulfolane as a glassing agent, limiting its application for invivo experiments. On the other hand, the BA-BDPA radical is soluble in PA and can be easily removed from the hyperpolarized solution by in-line filtration.

Herein, we report the synthesis and application as polarizing agents for dissolution DNP of two new BDPA derivatives: the benzyl

\footnotetext{
${ }^{a}$ Institut de Ciència de Materials de Barcelona, (ICMAB-CSIC) Campus Universitari de Bellaterra, E-08193 Cerdanyola del Vallès, Spain and Networking Research Center on Bioengineering, Biomaterials and Nanomedicine (CIBER-BBN), Barcelona, Spain.

${ }^{b}$ Servei de Ressonància Magnètica Nuclear, Facultat de Ciències i Biociències, Universitat Autònoma de Barcelona, E-08193 Bellaterra, Catalonia, Spain.

‡ These authors have contributed equally.

†Electronic Supplementary Information (ESI) available: [Synthesis and characterization of free radicals 1-7, EPR spectra of BAm-BDPA and CN-BDPA]. See DOI: $10.1039 / \mathrm{x} 0 \mathrm{xx} 00000 \mathrm{x}$
} 
amino BDPA (BAm-BDPA, 7, Chart 1) and the cyano (CN-BDPA, 5, Chart 1) radicals. Considering that a key step in dissolution DNP-NMR studies is the required polarization build-up time for an optimum signal enhancement, an experimental $\eta$ factor ( $\varepsilon$ /saturation time) to measure the efficiency of the dissolution DNP-NMR process has been introduced. The general polarizing behavior and efficiency of radicals 5 and 7 in front PA are analyzed and compared with the standards BDPA and OX63.

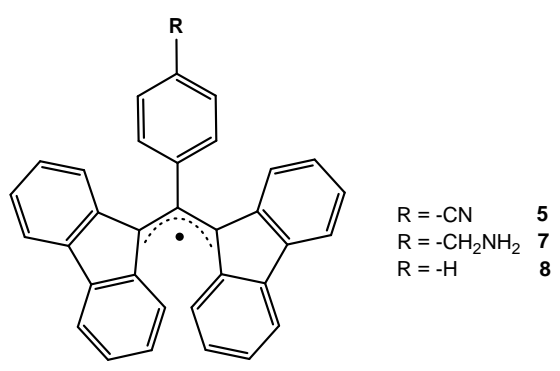

Chart 1 Structures of BDPA radical derivatives: CN-BDPA (5), BAm-BDPA (7) and the reference BDPA (8).

\section{Results and discussion}

\section{Synthesis and characterization}

The reference radical $\mathbf{8}$ is remarkably stable to oxygen in the solid state ${ }^{32}$ and has been reported to be stable to oxygen in solution with the exclusion of light. ${ }^{33}$ However, to achieve the highest persistance in solution in the dark it is necessary the presence of benzene which forms a complex with the radical. ${ }^{33}$ On the other hand, BDPA radical is reduced to give the corresponding carbanion when exposed to strong bases, such as hydroxide or alkoxide, and also reacts with strong acids. ${ }^{32}$

Radical 8 was firstly synthesized by the Koelsch's method. ${ }^{32}$ Then, Kuhn and Neugebeauer developed an alternative method which involved fewer steps. ${ }^{34}$ Efforts to synthesize BDPA derivatives have been carried out by few research groups, ${ }^{35,36}$ like Kuhn $^{34}$ and $\mathrm{Fox}^{33}$, and recently we have reported the synthesis of a benzyl alcohol derivative of BDPA (BA-BDPA). ${ }^{28}$ On the other hand, biradicals containing BDPA units have been limited only to molecules containing two BDPA radicals linked through the phenyl ring. ${ }^{34}$

In this work, the synthesis of the cyano BDPA derivative at the 4-position of the phenyl ring (4) was carried out following the Kuhn and Neugebeauer methodology, ${ }^{34}$ because it requires fewer steps and provides higher yields (Scheme 1 ). This multistep synthesis consisted on a condensation between fluorene and 4-cyanobenzaldehyde followed by a double bond bromination to obtain compound 2. The latest step was improved by changing the previously reported reaction conditions using sonication of suspended compound $\mathbf{1}$ in $\mathrm{CCl}_{4}$ for only five minutes. The next step was the elimination of hydrogen bromine to produce the conjugated acceptor $\mathbf{3}$, in a short reaction time (15 min.) to avoid the possible hydrolysis of the cyano group. Finally, the allylic substitution of $\mathbf{3}$ added the final fluorene ring that completes the BDPA structure (4). 


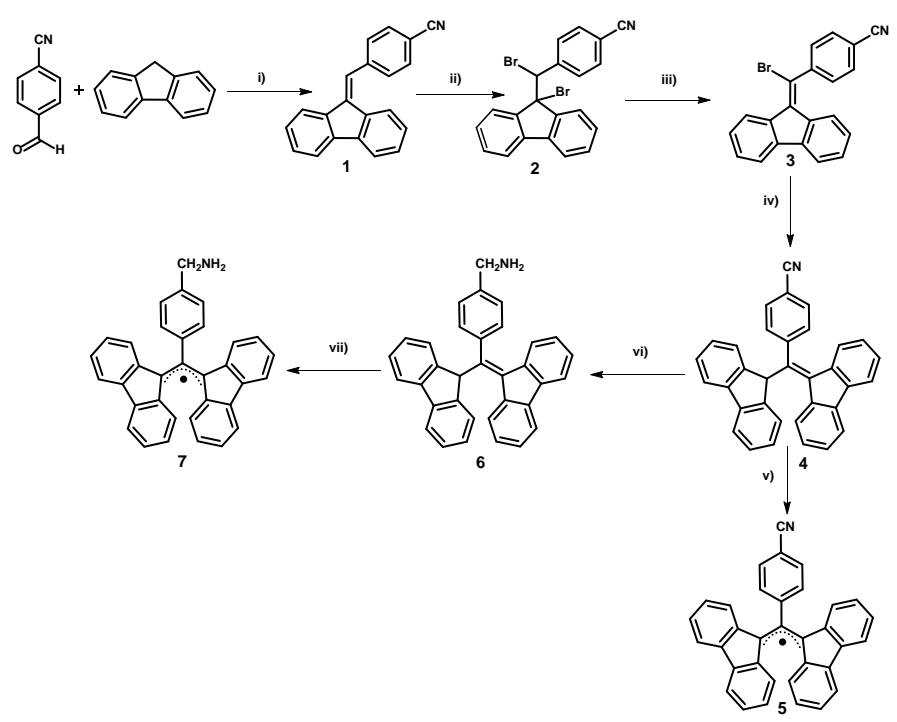

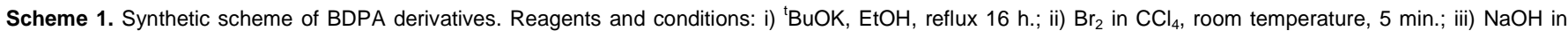
$\mathrm{EtOH}$, reflux, 30 min.; iv) Fluorene, ${ }^{\mathrm{t}} \mathrm{BuOK}$ in DMA room temperature, 15 min.; vi) $\mathrm{LiAlH}_{4} \mathrm{THF}$ anhydrous; v) and vii) $\mathrm{DBU}$ in DCM, then AgNO ${ }_{3}$.

By reduction of the cyano derivative 4 with $\mathrm{LiAlH}_{4}$ in anhydrous THF at low temperature, the benzylamino derivative 6 was isolated after treatment in acid media (Scheme 1). It appeared as a slightly red colored solid suggesting the presence of minimum concentrations of radical, as confirmed later on by the EPR spectrum. Although the sample contained such radical traces, the NMR spectra could be registered without problems.

Finally, radicals 5 and 7 were isolated by a treatment with 1,8-diazabicycloundec-7-ene (DBU), as a base, followed by AgNO , as an oxidant agent in dichloromethane (DCM). The total yields of 5 and 7 were $74 \%$ and $52 \%$, respectively. The carbanion formation and its oxidation to radical were monitored by UV-Vis due to the different absorbance of the anion and radical forms. BDPA carbanion derivatives show an absorption band at around $600 \mathrm{~nm}$ and the radical form at around $495 \mathrm{~nm}$. Thus, solutions of BDPA carbanion derivatives are in general blue colored, as is the case of radical 7, but in the case of radical 5 it was green due to the electron-withdrawing cyano group. However, the radical solutions were red in both cases, as for all reported BDPA radicals, because the electron density of the SOMO orbital is mainly located over the fluorenyl rings with null perturbation of the substituted phenyl ring. ${ }^{37,38}$ The EPR spectra at room temperature and in frozen solution of $\mathbf{5}$ and $\mathbf{7}$, as well as their corresponding simulations are depicted in Figs. S1-S3 of the ESI.

\section{Dissolution DNP studies using BAm-BDPA (7) and CN-BDPA (5) radical derivatives.}

Due to the insolubility of radical 5 in neat PA, the samples were prepared using sulfolane as a co-solvent, as previously described for the BDPA radical. ${ }^{31}$ Thus, once the radical 5 was completely dissolved in $100 \mu \mathrm{l}$ of sulfolane, the same amount of PA (100 $\mu$ ) was added, obtaining a final $40 \mathrm{mM}$ concentration of the radical in the mixture. The ${ }^{13} \mathrm{C}$ microwave DNP spectrum (Fig. S4a), acquired with $100 \mu \mathrm{l}$ of the previously prepared solution, showed a positive polarization peak at $\mathrm{P}(+)=94.080 \mathrm{GHz}($ frequency of work) and the negative one at $\mathrm{P}(-)=94.120 \mathrm{GHz}$. The polarization build-up curve (Fig. S4b) was performed with a $30 \mu \mathrm{l}$ aliquot of the frozen sample $(40 \mathrm{mM})$ irradiated at the optimal frequency. A slow polarization build-up curve with low polarization level was obtained, and, in addition, the sample was not stable with time (see ESI). Since the decomposition of the radical 5 could be the origin of such behavior, ${ }^{33}$ as occurs for the BDPA radical, several experiments with equimolar quantities of radical and benzene (see ESI) were repeated. However, the data obtained by the build-up curves were similar to those obtained in the previous case. Thus, it could be concluded that $\mathbf{5}$ is not a good polarizing agent of PA due to its low stability.

As noted before, radical BAm-BDPA 7 is soluble in neat PA enabling thereby its use without any glassing agent. The sample was prepared by dissolving 7 into PA and then an equimolar quantity of benzene was added giving a $40 \mathrm{mM}$ concentration of 7 . The acquisition of the ${ }^{13} \mathrm{C}$ microwave DNP spectrum was carried out with an aliquot of $50 \mu \mathrm{l}$ of the previous described sample and showed a positive polarization peak at $94.080 \mathrm{GHz}$ and a negative one at $94.125 \mathrm{GHz}$ (Fig. 1a). The separation between both polarization peaks $(|\mathrm{P}(+)-\mathrm{P}(-)|=94080$ - 94125) was $45 \mathrm{MHz}$, which is an intermediate value between the non-functionalized BDPA radical ${ }^{24}(50 \mathrm{MHz})$ and the cyano derivative $5(40 \mathrm{MHz})$. This could fulfill the requirements to polarize ${ }^{13} \mathrm{C}$ nuclei by the thermal mixing mechanism as commercial BDPA radical does. ${ }^{31}$ The optimal frequency of work was determined as the positive peak polarization of $\mathrm{P}(+)=94,080 \mathrm{GHz}$. 
Several samples were prepared $(20,40,60$ and $80 \mathrm{mM})$ to determine the optimal concentration of radical 7 for DNP experiments. The samples were made dissolving the appropriate quantity of radical 7 into PA and adding an equimolar quantity of benzene. A $20 \mu \mathrm{l}$ aliquot of each concentration was polarized at the optimal frequency until saturation, except for the $20 \mathrm{mM}$ sample. As can be observed in Fig. 1b, the best polarization level in solid-state conditions was achieved for the $60 \mathrm{mM}$ sample. The time needed to saturate the different samples was quite different depending on radical concentration, decreasing at higher concentrations. It could be observed how the curve corresponding to the $20 \mathrm{mM}$ is not saturated after $5400 \mathrm{~s}$ of polarization while the $60 \mathrm{mM}$ sample only needs $1800 \mathrm{~s}$ to reach the maximum solid-state polarization level. In the case of the most concentrated $80 \mathrm{mM}$ sample, saturation was achieved faster (1100 s) at expense to a slightly lower enhancement. These saturation times are also reflected as an exponential decay in the build-up time constants $\left(T_{c}\right.$, Fig. 2). The Tc value is lower at higher concentrations which could be associated to a thermal mixing mechanism. ${ }^{39}$
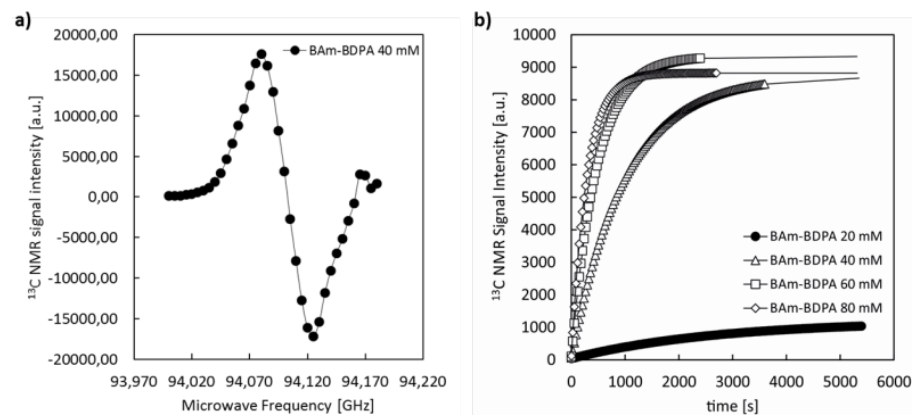

Fig. 1 a) ${ }^{13} \mathrm{C}$ microwave DNP spectrum of $50 \mu \mathrm{l}\left[1-{ }^{13} \mathrm{C}\right]$ pyruvic acid doped with $40 \mathrm{mM}$ radical 7 at $3.35 \mathrm{~T}$ and $1.4 \mathrm{~K}$. b) Solid-state ${ }^{13} \mathrm{C}$ polarization build-up curves of $20 \mu \mathrm{l}\left[1-{ }^{13} \mathrm{C}\right]$ pyruvic acid doped with different concentrations of radical 7.

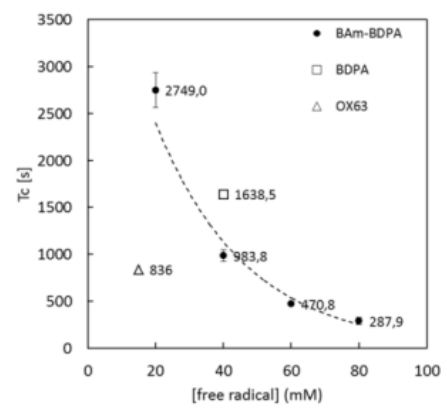

Fig. 2 Build-up time constants $\left(T_{c}\right)$ versus concentration of radical 7, OX63 and BDPA, performed at their optimal sample conditions in PA. Notice that BDPA includes sulfolane in a 1:1 ratio.

After polarization, all the samples were fast dissolved into hot water dissolution of $\mathrm{Na}_{2}$ EDTA (743 $\mu$ M) and automatically transferred to a $600 \mathrm{MHz}$ NMR spectrometer to perform the corresponding ${ }^{13} \mathrm{C}$ NMR measurements. The overall transfer time was $8.5 \mathrm{~s}$ (6 s of transfer between DNP and NMR instruments, $2 \mathrm{~s}$ of sample stabilization and 500 ms of measurement time). The liquid-state signal enhancement $(\varepsilon)$ was calculated by the acquisition of a conventional $1 \mathrm{D}{ }^{13} \mathrm{C}$ NMR experiment with a single scan and using an excitation flip angle $\theta=90^{\circ}$, for all four hyperpolarized and the thermal equilibrium samples (Entries 1-4, Table 1). To measure the thermal sample, the hyperpolarized sample was maintained 15 minutes outside the magnet to ensure the complete relaxation. It can be observed in Table 1 that the maximum signal enhancement in liquid-state conditions is achieved for the 60 $\mathrm{mM}$ sample, in strong agreement with the solid-state measurements.

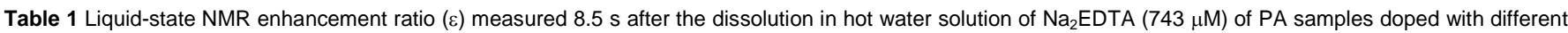
concentrations of radical 7, BDPA and OX63. $\%{ }^{13} \mathrm{C}$ polarization after sample dissolution was measured at $298 \mathrm{~K}$ into and a magnetic field of $14.1 \mathrm{~T}$.

\begin{tabular}{|c|c|c|c|c|c|c|}
\hline Entry & Radical & $\begin{array}{c}\text { Sat. } \\
\text { time }^{\mathrm{a}}[\mathrm{s}]\end{array}$ & $\begin{array}{c}\text { Pol. } \\
\text { time [s] }\end{array}$ & $\begin{array}{l}\text { Liquid-state } \\
\text { enhancement } \\
(\varepsilon)\end{array}$ & $\begin{array}{c}\text { \%pol } \\
{ }^{13} \mathrm{C} \\
\text { after } \\
\text { dissol. }\end{array}$ & $\begin{array}{c}\eta \\
\text { ( } / \text { sat. } \\
\text { time) } \\
{\left[\mathrm{s}^{-1}\right]}\end{array}$ \\
\hline
\end{tabular}




\begin{tabular}{|c|c|c|c|c|c|c|}
\hline 1 & $\begin{array}{l}\text { BAm- } \\
\text { BDPA } \\
20 \mathrm{mM}\end{array}$ & - & 5400 & 872 & 1.1 & -- \\
\hline 2 & $\begin{array}{l}\text { BAm- } \\
\text { BDPA } \\
40 \mathrm{mM}\end{array}$ & 3500 & 3600 & 7109 & 8.7 & 2.0 \\
\hline 3 & $\begin{array}{l}\text { BAm- } \\
\text { BDPA } \\
60 \mathrm{mM}\end{array}$ & 1800 & 2400 & 9358 & 11.4 & 5.2 \\
\hline 4 & $\begin{array}{l}\text { BAm- } \\
\text { BDPA } \\
80 \mathrm{mM}\end{array}$ & 1100 & 2600 & 6623 & 8.1 & 6.0 \\
\hline 5 & $\begin{array}{l}\text { BDPA } \\
40 \mathrm{mM}\end{array}$ & 5400 & 5400 & 11583 & 14.1 & 2.1 \\
\hline 6 & $\begin{array}{l}\text { BDPA } \\
40 \mathrm{mM}\end{array}$ & 5400 & 1800 & 4478 & 5.5 & 0.8 \\
\hline 7 & $\begin{array}{c}\text { OX63 } 15 \\
\mathrm{mM}\end{array}$ & 3600 & 3600 & 12369 & 15.1 & 3.4 \\
\hline 8 & $\begin{array}{c}\text { OX63 } 15 \\
\mathrm{mM}\end{array}$ & 3600 & 1800 & 7228 & 8.8 & 2.0 \\
\hline
\end{tabular}

a The saturation time has been taken as the time corresponding to the $98 \%$ solid-state polarization percentage estimated by the HyperSense ${ }^{\circledR}$ during the polarization build-up curve acquisition.

In order to compare these results with those with the non-functionalized BDPA radical and the golden standard OX63 radical, dissolution DNP experiments were also performed using these radicals in their optimal concentrations as was previously described: PA:sulfolane (1:1) doped with $40 \mathrm{mM}$ of BDPA and PA doped with $15 \mathrm{mM}$ of OX63. Fig. 3 shows the polarization build-up curves recorded in a HyperSense ${ }^{\circledR}$ polarizer working at $3.35 \mathrm{~T}$ and $1.4 \mathrm{~K}$, whereas Table 1 displays the corresponding liquid-state NMR signal enhancement factors. In addition, we introduce an efficient polarization factor ( $\eta$ ) to assess the liquidstate signal enhancement achieved by DNP saturation time afforded during the complete dissolution DNP-NMR process $(\eta=$ ع/saturation time [s], last column in Table 1).

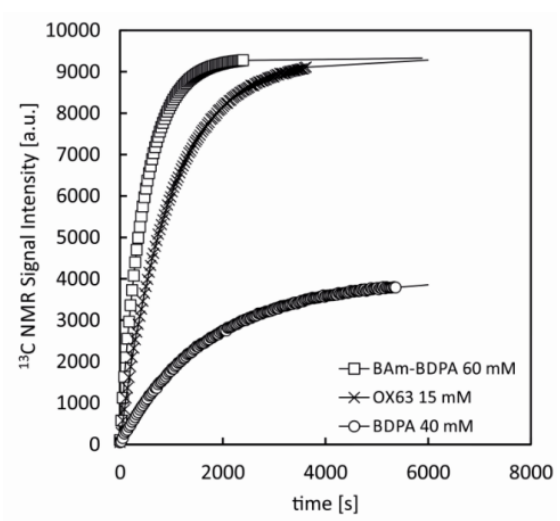

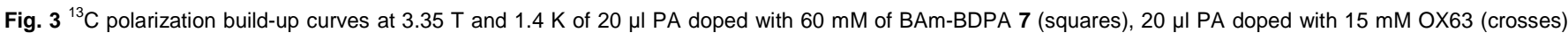

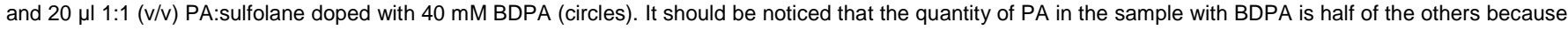
of its formulation.

As can be observed in Fig. 3, the polarization time required to reach the saturation plateau with a $60 \mathrm{mM}$ sample of 7 is two times lower than with OX63 radical and three times lower than with BDPA (Entries 3, 5 and 7 in Table 1). However, the $\varepsilon$ factor and the percentage of ${ }^{13} \mathrm{C}$ polarization are partially lower with respect to OX63 and BDPA radicals, mainly because the different radical concentrations could lead to different hyperpolarization decay rates. On the other hand, although $\varepsilon$ at a $80 \mathrm{mM}$ concentration is not too much high, we must not ignore that the saturation time is even shorter $(1100 \mathrm{~s}$; solid-state curve shown in Fig. $1 \mathrm{~b}$, Entry 4 in 
Table 1), specifically more than three times lower than OX63 and five times lower than BDPA. Thus, although the $\varepsilon$ at both 60 $\mathrm{mM}$ and $80 \mathrm{mM}$ show partially lower enhancements, the use of radical 7 as polarizing agent seems to be advantageous since it can reduce the overall experimental time with respect to OX63 and BDPA radicals, resulting in a very efficient polarizing agent. This is clearly observed comparing the different $\eta$ factors in Table 1 (Entries 3, 5 and 7). Typically, the time needed to polarize a sample in a dissolution DNP experiment using the OX63 trityl radical is 60-90 min. whereas with for a $60 \mathrm{mM}$ solution of 7 it can be significantly reduced to $30 \mathrm{~min}$. This leads to economize instrumentation time and minimize the consumption of liquid helium. Comparison of build-up time constant $\left(\mathrm{T}_{\mathrm{c}}\right)$ of BAm-BDPA 7 with the ones for OX63 and BDPA radicals performed at their optimal concentration in PA (Fig. 2) shows the same tendency. Indeed, the build-up time constant for radical 7 is lower than for OX63 and BDPA radicals, and hence the polarization time needed to polarize PA is also inferior.

Taking into account the ${ }^{13} \mathrm{C}$ polarization build-up curves at solid-state (Fig. 3), a comparison of the liquid-state DNP enhancements of all three samples at short polarization times was performed. Thus, after $1800 \mathrm{~s}$ of polarization, all the samples were fast dissolved into hot water dissolution of $\mathrm{Na}_{2}$ EDTA $(743 \mu \mathrm{M})$ and automatically transferred to a $600 \mathrm{MHz}$ NMR spectrometer to perform the corresponding NMR measurements (Entries 3, 6 and 8 in Table 1). It is worth noting that the highest signal enhancement $(\varepsilon)$ is obtained by $7(60 \mathrm{mM})$, which is 1.3 and 2 times higher than with OX63 and BDPA radicals, respectively. We could also observe that $\mathbf{7}$ is the most efficient polarizing agent as it has the highest $\eta$ factor meaning an optimal compromise between a reasonable signal enhancement and a relatively short polarization time.

\section{Conclusions}

Two novel BDPA radical derivatives have been synthesized and studied as polarizing agents for fast dissolution DNP-NMR applications. The radical CN-BDPA, 5, has shown to be unstable with time and hence it is not a good polarizing agent of PA. On the other hand, the radical BAm-BDPA, 7, has proved to be a quick and efficient polarizing agent of PA without need of glassing agent, showing optimum levels of ${ }^{13} \mathrm{C}$ signal NMR enhancements per polarization time unit. Its use as polarizing agent in dissolution DNP studies can represent important saves in measurement time, optimized instrument usage and liquid helium consumption.

\section{Material and methods}

Tris \{8-carboxyl-2,2,6,6-tetra[2-(1-hydroxyethyl)]-benzo(1,2-d:4,5-d')bis(1,3)dithiole-4-yl\}methyl sodium salt (OX63, Oxford Instruments Molecular Biotools, Oxford, UK), 1,3-bisdiphenylene-2-phenylallyl (BDPA) radical/benzene clathrate (1:1), [1-

${ }^{13} \mathrm{C}$ ]pyruvic acid, sulfolane and all the solvents were purchased from Sigma-Aldrich Company, Ltd. (Tres Cantos, Madrid, Spain) and were used without further purification.

\section{X-band EPR}

The EPR spectra were recorded in a Bruker ELEXYS E500 X-band spectrometer equipped with a TE102 microwave cavity, a Bruker variable temperature unit, a field-frequency (F/F) lock system Bruker ER 033 M; line positions were determined with an NMR Gaussmeter Bruker ER 035 M. The modulation amplitude was kept well below the line width, and the microwave power was well below saturation.

Dynamic Nuclear Polarization Sample Preparations: (a) Radical 5 solutions: $3.54 \mathrm{mg}$ of radical 5 were dissolved in 100 ul of sulfolane and then the same amount of PA (100 $\mu \mathrm{l})$ was added, obtaining a final $40 \mathrm{mM}$ concentration of the radical in the mixture. After the first trial: $3.54 \mathrm{mg}$ were dissolved in $100 \mu \mathrm{l}$ of sulfolane and benzene ( $80 \mathrm{mM})$. Once the radical was completely dissolved, $100 \mu \mathrm{l}$ of PA were added, obtaining a final solution where the concentration of both radical and benzene was 40 mM. (b) Radical 7 solutions: $1.8 \mathrm{mg}$ of 7 were completely dissolved in $100 \mu \mathrm{l}$ of PA and $0.36 \mu$ l of benzene were added. The final concentration of both 7 and benzene was $40 \mathrm{mM}$. The same protocol was followed in order to prepare solutions of 7 at all different concentrations (20, 60 and $80 \mathrm{mM}$ ). (c) BDPA 8 solutions: $40 \mathrm{mM}$ BDPA solutions were prepared by dissolving $4.0 \mathrm{mg}$ of 8 into $100 \mu \mathrm{l}$ of sulfolane, then $100 \mu \mathrm{l}$ of PA were added yielding the desired concentration. (d) OX63 solutions: $15 \mathrm{mM}$ solutions of OX63 were prepared by completely dissolving $2.4 \mathrm{mg}$ of radical into $100 \mu \mathrm{l}$ of PA.

Microwave sweep: The optimum positive $\mathrm{P}(+)$ and negative $\mathrm{P}(-)$ polarization peaks for each radical sample were determined by irradiating for 3 minutes at each frequency in a range from 94.000 to $94.200 \mathrm{GHz}$ unless otherwise is stated.

Dissolution DNP Experiments: A HyperSense ${ }^{\circledR}$ (Oxford Instruments Molecular Biotools, Oxford, UK) commercial polarizer working at $3.35 \mathrm{~T}$ and $1.4 \mathrm{~K}$ was used to hyperpolarize $20 \mu \mathrm{l}$ aliquots of each different sample. These were polarized by irradiation at the corresponding optimum positive peak $\mathrm{P}(+)$ determined by the ${ }^{13} \mathrm{C}$ microwave DNP spectrum for each radical used until polarization saturation. The hyperpolarized sample was then dissolved with $4 \mathrm{ml}$ water and automatically transferred into an empty NMR tube placed inside a Bruker AVANCE-III $600 \mathrm{MHz}$ spectrometer (Bruker Biospin, Rheinstetten, Germany), previously locked and shimmed with a reference sample. The spectrometer was equipped with a 5 mm broadband TXI inverse probehead incorporating a z-gradient coil and working at field strength of $14.1 \mathrm{~T}\left(600.13\right.$ and $150.03 \mathrm{MHz}$, ${ }^{1} \mathrm{H}$ and ${ }^{13} \mathrm{C}$ frequencies 
respectively). After dissolution, the polytetrafluoroethylene (PTFE) transfer pipe line was cleaned with with methanol (3 $\mathrm{x} 4 \mathrm{ml}$ ) and then with Water/ $\mathrm{Na}_{2}$ EDTA $(743 \mu \mathrm{M})(3 \mathrm{x} 4 \mathrm{ml})$, after each DNP experiment.

Liquid-state ${ }^{13} \mathrm{C}$ DNP-NMR measurements: The ${ }^{13} \mathrm{C}$ DNP-NMR spectra were acquired $8.5 \mathrm{~s}$ after the sample dissolution using a conventional $1 \mathrm{D}$ broadband, heterodecoupled ${ }^{13} \mathrm{C}$ spectrum was recorded employing a $90^{\circ} \mathrm{RF}$ pulse. The data were collected into $32 \mathrm{~K}$ data points during an acquisition time of $0.43 \mathrm{~s}$ and using a spectral width of $37878 \mathrm{~Hz}$ in a single scan. Prior to Fourier transformation an exponential function with a line broadening of $1 \mathrm{~Hz}$ was applied, the frequency-domain spectra were then manually phased and baseline corrected.

After complete decay of polarization, a conventional $1 \mathrm{D}{ }^{13} \mathrm{C}$ NMR thermal equilibrium spectrum was acquired with an acquisition time of $0.43 \mathrm{~s}$. The data were collected into $32 \mathrm{~K}$ data points, with a spectral width of $37878 \mathrm{~Hz}$ in a single and using a $90^{\circ}$ degree flip angle, unless otherwise stated. It was processed in the same way than hyperpolarized ${ }^{13} \mathrm{C}$ DNP-NMR spectrum. The liquidstate enhancements were calculated, by the division of the signal integral of the hyperpolarized signal over the integral of the thermal equilibrium.

\section{Acknowledgements}

This research was supported by the DGI Grants "POMAs" (CTQ2010-19501) and "Be-Well" (CTQ2013-40480-R), MINECO (CTQ2012-32436), AGAUR Grant (2014SGR-0017) and MINECO Grant (PTA 2011-5037-I) for the subprogram Personal Técnico de Apoyo. We also thank the Networking Research Center on Bioengineering, Biomaterials and Nanomedicine (CIBERBBN, Consejo Superior de Investigaciones Científicas by the JAE Grant, Amable Bernabé for his work in MALDI spectrometry. NMR studies were carried out at the joint NMR facility of the Universitat Autònoma de Barcelona and CIBER-BBN (Cerdanyola del Vallès).

TOC

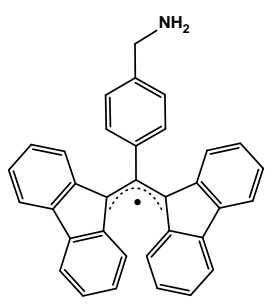

BAm-BDPA

DNP saturation time: 1800 s

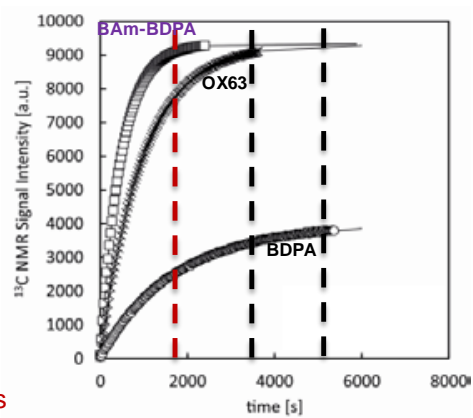




\section{Notes and references}

${ }^{1}$ J. Kurhanewicz, D. B. Vigneron, K. Brindle, E. Y. Chekmenev, R. J. Deberardinis, G. G. Green, R. R. Rizi, B. D. Ross and W. S. Warren, Neoplasia, $2011,13,81$.

2 J.H. Lee, Y. Okuno, and S. Cavagnero, J. Magn. Reson., 2014, 241, 18.

${ }^{3}$ F. Colegrove, L. Schearer and G. Walters, Phys. Rev., 1963, 132, 2561.

${ }^{4}$ H.-U. Kauczor, R. Surkau and T. Roberts, Eur. Radiol., 1998, 8, 820.

${ }^{5}$ S. B. Duckett and R. E. Mewis, Acc. Chem. Res., 2012, 45, 1247.

${ }^{6}$ C. Bowers and D. Weitekamp, Phys. Rev. Lett., 1986, 57, 2645.

${ }^{7}$ A. Abragam and M. Goldman, Reports Prog. Phys., 1978, 41, 395.

${ }^{8}$ K. H. Sze, Q. Wu, H. S. Tse and G. Zhu, Top. Curr. Chem., 2012, 326, 215.

${ }^{9}$ J. H. Ardenkjær-Larsen, B. Fridlund, A. Gram, G. Hansson, L. Hansson, M. H. Lerche, R. Servin, M. Thaning and K. Golman, Proc. Natl. Acad. Sci. U. S. A., 2003, $100,10158$.

${ }^{10}$ F. A. Gallagher, M. I. Kettunen and K. M. Brindle, Prog. Nucl. Magn. Reson. Spectrosc., 2009, 55, 285.

${ }^{11}$ H. Kovacs, D. Moskau and M. Spraul, Prog. Nucl. Magn. Reson. Spectrosc., 2005, 46, 131.

${ }^{12}$ M. Ragavan, H.-Y. Chen, G. Sekar and C. Hilty, Anal. Chem., 2011, 83, 6054.

${ }^{13}$ S. Bowen and C. Hilty, Angew. Chem. Int. Ed. Engl., 2008, 47, 5235.

${ }^{14}$ H. Zeng, Y. Lee and C. Hilty, Anal. Chem., 2010, 82, 8897.

${ }^{15}$ M. H. Lerche, S. Meier, P. R. Jensen, H. Baumann, B. O. Petersen, M. Karlsson, J. O. Duus and J. H. Ardenkjaer-Larsen, J. Magn. Reson., $2010,203,52$.

${ }^{16}$ L. Frydman and D. Blazina, Nat. Phys., 2007, 3, 415.

${ }^{17}$ P. Giraudeau, Y. Shrot and L. Frydman, J. Am. Chem. Soc., 2009, 131, 13902.

${ }^{18}$ M. E. Merritt, C. Harrison, C. Storey, F. M. Jeffrey, D. Sherry and C. R. Malloy, Proc. Natl. Acad. Sci. U. S. A., 2007, 104, 19773.

${ }^{19}$ K. M. Brindle, S. E. Bohndiek, F. A. Gallagher and M. I. Kettunen, Magn. Reson. Med., 2011, 66, 505.

${ }^{20}$ M. Karlsson, P. R. Jensen, J. Ø. Duus, S. Meier and M. H. Lerche, Appl. Magn. Reson., 2012, 43, 223.

${ }^{21}$ L. Chavarria, J. Romero-Giménez, E. Monteagudo, S. Lope-Piedrafita and J. Cordoba, NMR Biomed. 2015, 28, 17.

${ }^{22}$ W. Jiang, L. Lumata, W. Chen, S. Zhang, Z. Kovacs, A. D. Sherry and C. Khemtong, Sci. Rep., 2015, 5, 9104.

${ }^{23}$ D. R. Anderson, J. S. Keute, H. L. Chapel and T. H. Koch, J. Am. Chem. Soc., 1979, 101, 1904.

${ }^{24}$ L. Lumata, M. E. Merritt, C. R. Malloy, A. D. Sherry and Z. Kovacs, J. Phys. Chem. A, 2012, 116, 5129.

${ }^{25}$ (a) C. Rovira, D. Ruiz-Molina, O. Elsner, J. Vidal-Gancedo, J. Bonvoisin, J.-P. Launay and J. Veciana, Chem. Eur. J., 2001, 7, 240. (b) D. Maspoch, D. RuizMolina, K. Wurst, J. Vidal-Gancedo, C. Rovira and J. Veciana, Dalton Trans., 2004, 7, 1073. (c) V. Lloveras, J. Vidal-Gancedo, T. M. Figueira-Duarte, J.-F. Nierengarten, J. J. Novoa, F. Mota, N. Ventosa, C. Rovira and J. Veciana, J. Am. Chem. Soc., 2011, 133, 5818.

${ }^{26}$ (a) J. L. Muñoz-Gomez, I. Marin-Montesinos, V. Lloveras, M. Pons, J. Vidal-Gancedo and J. Veciana, Org. Lett., 2014, 16, 5402. (b) C. Gabellieri, V. Mugnaini, P. C. Paniagua, N. Roques, M. Oliveros, M. Feliz, J. Veciana and M. Pons, Angew. Chem. Int. Ed., 2010, 49, 3360. (c) C. Ysacco, E. Rizzato, M. A. Virolleaud, H. Karoui, A. Rockenbauer, F. Moigne, D. Siri, O. Ouai, R. G. Griffin and P. Tordo, Phys. Chem. Chem. Phys., 2010, 12, 5841. (d) E. L. Dane, B. Corzilius, E. Rizzato, P. Stocker, T. Maly, A. A. Smith, R. G. Griffin, O. Ouai, P. Tordo and T. M. Swager, J. Org. Chem., 2012, 77, 1789. (e) O. Haze, B. Corzilius, A. A. Smith, R. G. Griffin and T. M. Swager, J. Am. Chem. Soc., 2012, 134, 14287.

${ }^{27}$ J. H. Ardenkjaer-Larsen, I. Laursen, I. Leunbach, G. Ehnholm, L.-G. Wistrand, J. S. Petersson and K. Golman, J. Magn. Reson., $1998,133,1$.

${ }^{28}$ J. L. Muñoz-Gómez, E. Monteagudo, V. Lloveras, T. Parella, J. Veciana and J. Vidal-Gancedo, Org. Biomol. Chem., 2015, 13, 2689.

${ }^{29}$ V. K. Michaelis, A. A. Smith, B. Corzilius, O. Haze, T. M. Swager and R. R. Griffin, J. Am. Chem. Soc., 2013, 135, 2935.

${ }^{30}$ L. Becerra, G. Gerfen, R. Temkin, D. Singel and R. Griffin, Phys. Rev. Lett., 1993, 71, 3561.

${ }^{31}$ L. Lumata, S. J. Ratnakar, A. Jindal, M. Merritt, A. Comment, C. Malloy, A. D. Sherry and Z. Kovacs, Chem. Eur. J., 2011, $17,10825$.

${ }^{32}$ C. F. Koelsch, J. Am. Chem. Soc., 1957, 79, 1165.

${ }^{33}$ D.T. Breslin and M. A. Fox, J. Phys. Chem., 1993, 97, 13341.

${ }^{34}$ R. Kuhn and F. A. Neugebeauer, Monatsh. Chem., 1964, 95, 3.

${ }^{35}$ M. J. Plater, S. Kemp and E. Lattmann, J. Chem. Soc. Perkin Trans. 1, 2000, 971.

${ }^{36}$ N. Hiroyuki, Y. Naoki, S. Yukoh, G. Ritsuko, M. Takeshi and T. Eishun, J. Macromol. Sci., Part A, 1992, $29,775$.

${ }^{37}$ S. Mullegger, M. Rashidi, M. Fattinger and R. Koch, J. Phys. Chem. C, 2012, 116, 22587.

${ }^{38}$ N. Azuma, T. Ozawa, and J. Yamauchi., J. Chem. Soc., Perkin Trans. 2, 1994, 203.

${ }^{39}$ L. Lumata, A. K. Jindal, M. E. Merritt, C. R. Malloy, A. D. Sherry and Z. Kovacs, J. Am. Chem. Soc., 2011, 133, 8673-8680. 